\title{
Coupled Canonical Grid/Discrete Dipole Approach for Computing Scattering from Objects Above or Below a Rough Interface
}

\author{
Joel T. Johnson, Member, IEEE, and Robert J. Burkholder, Senior Member, IEEE,
}

\begin{abstract}
A numerical model for computing scattering from a three-dimensional (3-D) dielectric object above or below a rough interface is described. The model is based on an iterative method of moments solution for equivalent electric and magnetic surface current densities on the rough interface and equivalent volumetric electric currents in the penetrable object. To improve computational efficiency, the canonical grid method and the discrete dipole approach (DDA) are used to compute surface to surface and object to object point couplings, respectively, in $O(N \log N)$, where $N$ is the number of surface or object sampling points. Two distinct iterative approaches and a preconditioning method for the resulting matrix equation are discussed, and the solution is verified through comparison with a Sommerfeld integral-based solution in the flat surface limit. Results are illustrated for a sample landmine detection problem and show that a slight surface roughness can modify object backscattering returns.
\end{abstract}

Index Terms-Electromagnetic scattering, ground penetrating radar (GPR), radar cross section, rough surface scattering.

\section{INTRODUCTION}

$\mathbf{E}$ LECTROMAGNETIC scattering from objects is affected by the surrounding medium. Many realistic geometries involve objects in the presence of the ground, which is often modeled as a planar dielectric boundary. However, roughness on the ground surface can potentially modify object scattering returns from those with a flat surface, particularly in cases where the roughness size becomes larger than a fraction of the electromagnetic wavelength. Analysis of these problems is complicated by the many possible scattering interactions between the rough surface and object; at present, approximate analytical solutions exist only in the small roughness limit [1]-[4]. The development of numerical models is therefore of interest since these models avoid any approximations and thus can clarify important scattering effects when analytical methods fail. The fact that a combined object/rough surface scattering model can be applied to

\footnotetext{
Manuscript received September 30, 2000; revised December 15, 2000. This work was supported by ONR Contracts N00014-97-1-0541 and N00014-00-10399, NSF Project ECS-9701678, and by a grant from Duke University as part of the OSD MURI on Humanitarian Demining. Use of the IBM SP system at the Maui High Performance Computing Center is acknowledged and was sponsored by the Air Force Research Laboratory, Air Force Material Command under cooperative Agreement F29601-93-2-0001. Opinions, interpretations, conclusions, and recommendations are those of the authors and are not necessarily endorsed by the U.S. Air Force, Air Force Research Laboratory, or the U.S. Government.

The authors are with the Department of Electrical Engineering and ElectroScience Laboratory, Ohio State University, Columbus, OH 43210 USA.

Publisher Item Identifier S 0196-2892(01)04841-0.
}

studies of landmine detection [5], foliage and surface remote sensing [2], or target detection and signal processing problems [6] provides further motivation for model development.

Several previous numerical studies of scattering from objects in the presence of the ground have been reported. For geometries with a flat surface model, a Sommerfeld Green's function can be derived for frequency domain methods, and discretization is required only on the object [7], [8]. Time domain studies of objects in the presence of flat boundaries have also been reported [9]. Previous studies including objects and surface roughness have primarily been limited to one-dimensional (1-D) surface geometries (i.e., surfaces having roughness only in one horizontal direction) [10]-[13] since surface unknowns must be included and computational requirements are increased. Problems involving two-dimensional (2-D) surfaces and 3-D objects have been considered only in a small number of studies [6], [14] due to the large computational requirements, and have been limited in most cases to perfectly conducting objects.

In this paper, an efficient numerical model is described which makes studies of scattering from a 3-D dielectric object in the presence of a 2-D rough surface possible. The model is similar to that used in the perfectly conducting object study of [6], and is based on an iterative method of moments solution. The integral equation formulation and resulting matrix equation are presented in Section II. Iterative solutions of the matrix equation based on a standard nonstationary algorithm [15] or based on the "multiple interaction" iteration of [6] are then discussed in Section III. Efficiency of the iterative solution is improved through use of the "canonical grid" (CAG) method [16]-[22] and the discrete-dipole approximation (DDA) [23], [24] for computing surface to surface and object to object point couplings, respectively, as described in Section IV. A sample application of the model to studies of scattering from a subsurface object is then illustrated in Section V, and final conclusions presented in Section VI.

\section{FORMULATION}

Fig. 1 illustrates the geometry considered: a dielectric object with relative permittivity $\epsilon_{3}$ is located in the presence (in this case, below) of a rough interface $z=f(x, y)$ between Regions 1 (relative permittivity $\epsilon_{1}$ ) and 2 (relative permittivity $\epsilon_{2}$ ). Integral equations to determine time harmonic fields scattered in this problem can be formulated in terms of equivalent electric and magnetic surface current densities on the interface and equivalent electric volumetric currents in the object. 


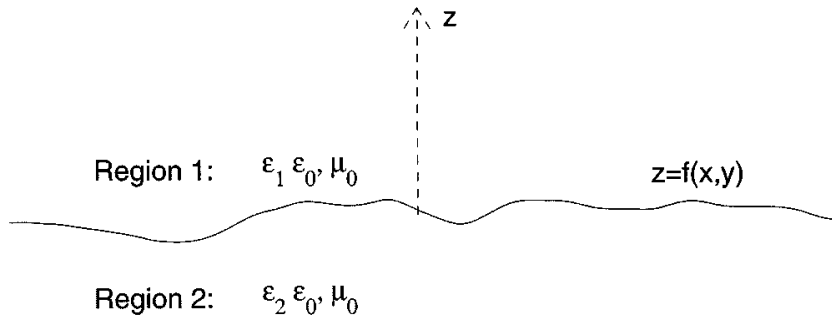

Object $\varepsilon_{3} \varepsilon_{0}, \mu_{0}$

Fig. 1. Geometry of problem: object may be above or below boundary.

\section{A. Surface Integral Equations}

For convenience, electric and magnetic surface current densities on the interface are defined here as $\bar{F}=\eta_{1} \bar{N} \times \bar{H}_{1}$ and $\bar{G}=-\bar{N} \times \bar{E}_{1}$, respectively, where $\bar{E}_{1}$ and $\bar{H}_{1}$ are the total electric and magnetic fields in Region 1 on the interface, $\eta_{1}$ is the characteristic impedance of Region 1 , and $\bar{N}$ is an upward pointing normal vector $\hat{z}-\hat{x}(\partial f / \partial x)-\hat{y}(\partial f / \partial y)$. Following the formulation of [25] and assuming an $e^{-i \omega t}$ time dependence, integral equations for $\bar{F}$ and $\bar{G}$ are

$$
\begin{aligned}
\frac{\bar{F}}{2}= & \bar{N} \times \iint_{A} d x^{\prime} d y^{\prime} \\
& \cdot\left\{i k_{1} g_{1} \bar{G}+\nabla g_{1} \times \bar{F}-\left(\frac{\nabla g_{1}}{i k_{1}}\right)\left|\bar{N}^{\prime}\right|\left[\nabla^{\prime} \cdot\left(\frac{\bar{G}}{\left|\bar{N}^{\prime}\right|}\right)\right]\right\} \\
& +\eta_{1} \bar{N} \times \bar{H}_{i n c, 1} \\
-\frac{\bar{G}}{2}= & -\bar{N} \times \iint_{A} d x^{\prime} d y^{\prime} \\
& \cdot\left\{i k_{1} g_{2} \bar{F}-\nabla g_{2} \times \bar{G}-\left(\frac{\nabla g_{2}}{i k_{1}}\right)\left|\bar{N}^{\prime}\right|\left[\frac{\epsilon_{1}}{\epsilon_{2}} \nabla^{\prime} \cdot\left(\frac{\bar{F}}{\left|\bar{N}^{\prime}\right|}\right)\right]\right\} \\
& +\bar{N} \times \bar{E}_{i n c, 2}
\end{aligned}
$$

where $k_{j}=k_{0} \sqrt{\epsilon_{j}}$ for $j=1 \cdots 3$ is the wavenumber in Region $j ; k_{0}=\omega \sqrt{\mu_{0} \epsilon_{0}}=(2 \pi / \lambda)$ is the free-space wavenumber for electromagnetic wavelength $\lambda$ and radian frequency $\omega$; and $g_{j}$ is the free-space Green's function in Region $j$

$$
g_{j}\left(\bar{r}, \bar{r}^{\prime}\right)=\frac{e^{i k_{j}\left|\bar{r}-\bar{r}^{\prime}\right|}}{4 \pi\left|\bar{r}-\bar{r}^{\prime}\right|}
$$

where $\nabla$ and $\nabla^{\prime}$ denote the gradient operator in terms of unprimed (observation) and primed (source) coordinates, respectively, and the integration is over the horizontal projected area $A$ of surface $z=f(x, y)$. The incident fields $\bar{H}_{i n c, 1}$ and $\bar{E}_{i n c, 2}$ describe fields which impinge from above or below the boundary, respectively, and will include contributions from the fields $\bar{E}_{\text {inc }}$ and $\bar{H}_{\text {inc }}$ which excite the problem (assumed to approach from Region 1) as well as the dielectric object. Use of the Stratton-Chu form and surface divergence operators in (1) and (2) results in four scalar unknown functions (i.e., the $x$ and $y$ components of $\bar{F}$ and $\bar{G}$, from which the $z$ components can be determined) to be found, as opposed to the six unknown scalar function formulations which have been used previously [6], [18]. A standard point matching discretization (i.e., surface currents assumed constant over a surface patch and integral equations "tested" at a set of points) is applied to these equations as described in [16]-[22], [25], so that surface current divergences $\nabla^{\prime} \cdot\left(\bar{F} /\left|\bar{N}^{\prime}\right|\right)$ and $\nabla^{\prime} \cdot\left(\bar{G} /\left|\bar{N}^{\prime}\right|\right)$ can be computed numerically using a centered difference method. Examination of self terms in (1) and (2) (i.e., when source and observation points overlap) shows cancellation of all terms involving $\nabla g_{j}$ for surface patches modeled locally as tilted planes due to symmetries in the integration. Singularities in the $g_{j}$ terms are integrable, and calculated efficiently through standard analytical subtraction/addition methods.

\section{B. Object Integral Equation}

A dielectric object in Region $j$ is replaced by an equivalent electric volumetric current density $\bar{J}$, for which the resulting integral equation is

$$
\begin{aligned}
\bar{E}= & \bar{E}_{i n c, v}+\iiint_{\text {object }} d V^{\prime} i \omega \mu_{0} g_{j} \\
& \cdot\left[\overline{\bar{I}}-\hat{R} \hat{R}+\frac{i k_{j} R-1}{k_{j}^{2} R^{2}}(\overline{\bar{I}}-3 \hat{R} \hat{R})\right] \cdot \bar{J}
\end{aligned}
$$

where

$$
\begin{array}{ll}
R & \left|\bar{r}-\bar{r}^{\prime}\right| ; \\
\hat{R} & \left(\left(\bar{r}-\bar{r}^{\prime} /\left(\left|\bar{r}-\bar{r}^{\prime}\right|\right) ;\right.\right.
\end{array}
$$

$\overline{\bar{I}} \quad$ unit dyad;

$\bar{E}_{\text {inc, } v}$ field which impinges upon the object including contributions from electric and magnetic surface current densities on the interface $z=f(x, y)$ and the field which excites the problem if the object is located in Region 1.

In a point discretization of $\bar{J}$ with uniform volume $\Delta V$, (4) can also be expressed in terms of a set of "dipole moments" $\bar{P}=$ $\Delta V \bar{J}\left(i / 4 \pi \epsilon_{j} \epsilon_{0} \omega\right)$, leading to a "discrete dipole" representation of equivalent currents [23]. Equation (4) involves three scalar unknown functions: the $\hat{x}, \hat{y}$, and $\hat{z}$ components of $\bar{J}$. Self terms in this formulation are described in [23], and replace the electric field on the left-hand side of (4) with $\bar{P} / \alpha$ where $\alpha$ is given by

$$
\alpha=\frac{3 \Delta V}{4 \pi}\left(\frac{\frac{\epsilon_{3}}{\epsilon_{j}}-1}{\frac{\epsilon_{3}}{\epsilon_{j}}+2}\right) .
$$

Note that inhomogeneous objects can also be included in the present formulation simply by varying values of $\epsilon_{3}$ and $\alpha$ for points in the object; homogeneous objects are considered in the results shown for simplicity.

\section{Surface and Object Coupling}

Coupling between surface and object is computed using formulations similar to (1), (2), and (4). For an object in Region 2 , coupling from surface sources to object observation points is calculated from

$$
\begin{aligned}
\bar{E}_{i n c, v}= & -\iint_{A} d x^{\prime} d y^{\prime} i k_{1} g_{2} \\
& \cdot\left[\overline{\bar{I}}-\hat{R} \hat{R}+\frac{i k_{2} R-1}{k_{2}^{2} R^{2}}(\overline{\bar{I}}-3 \hat{R} \hat{R})\right] \cdot \bar{F} \\
& +\iint_{A} d x^{\prime} d y^{\prime} g_{2} \frac{i k_{2} R-1}{R}(\hat{R} \times \bar{G})
\end{aligned}
$$


and coupling from object sources to surface observation points is found by evaluating $\bar{E}_{i n c, 2}$ from the integral term in (4) with $k_{j}=k_{2}$. For an object in Region 1, surface source to object coupling is obtained from

$$
\begin{aligned}
\bar{E}_{i n c, v}= & \bar{E}_{i n c}+\iint_{A} d x^{\prime} d y^{\prime} i k_{1} g_{1} \\
& \cdot\left[\bar{I}-\hat{R} \hat{R}+\frac{i k_{1} R-1}{k_{1}^{2} R^{2}}(\bar{I}-3 \hat{R} \hat{R})\right] \cdot \bar{F} \\
& -\iint_{A} d x^{\prime} d y^{\prime} g_{1} \frac{i k_{1} R-1}{R}(\hat{R} \times \bar{G})
\end{aligned}
$$

where $\bar{E}_{\text {inc }}$ is the electric field which excites the problem. Object source to surface coupling is found from

$$
\bar{H}_{i n c, 1}=\bar{H}_{i n c}+\iiint_{\text {object }} d V^{\prime} g_{1} \frac{i k_{1} R-1}{R}(\hat{R} \times \bar{J})
$$

where $\bar{H}_{\text {inc }}$ is the magnetic field which excites the problem corresponding to $\bar{E}_{\text {inc }}$.

In the results to be shown, object and surface points do not overlap, so that no singular terms in the coupling equations are encountered. For geometries which do contain overlapping points, singularities can be extracted and integrated by the methods described for (1), (2), and (4), although this has not been performed in the current implementation.

\section{Combined Matrix Equation}

Combining the surface, object, and coupling equations results in a matrix equation in terms of $\bar{F}, \bar{G}$, and $\bar{J}$. For an object in Region 2, the matrix equation can symbolically be written as

$$
\begin{aligned}
& {\left[\begin{array}{c|r}
\text { Surface : Surface } & \text {-Object : Surface } \\
\text {-Surface : Object } & \text { Object : Object }
\end{array}\right]} \\
& \cdot\left[\begin{array}{c}
\bar{F}, \bar{G} \\
\bar{J}
\end{array}\right]=\left[\begin{array}{c}
\bar{Q}_{i n c} \\
0
\end{array}\right]
\end{aligned}
$$

where "Surface: Surface" block represents minus the integral terms on the right-hand sides (RHSs) of (1) and (2). The "Object: Object" block represents minus the integral term on the RHS of (4), and "Object : Surface" and "Surface : Object" blocks are derived from integral terms of the equations described in Section II-C [written as negative since they originally appear on the RHSs of (1), (2), and (4)]. The case for an object in Region 1 is similar and will not be separated in the following discussions.

The "Surface: Surface" and "Object: Surface" blocks of the matrix equation are ordered so that the $\hat{x}$ and $\hat{y}$ components of (1) for a single observation point are following by the $\hat{x}$ and $\hat{y}$ components of (2); this arrangement places the $\pm 1 / 2$ self contributions on the diagonal of the "Surface: Surface" component when the $\bar{F}$ and $\bar{G}$ unknowns are ordered as $F_{x}, F_{y}, G_{x}$, and $G_{y}$ for a given source point in the unknown vector. The RHS $\bar{Q}_{i n c}$ thus is ordered as $\hat{x} \cdot \eta_{1} \bar{N} \times \bar{H}_{i n c}, \hat{y} \cdot \eta_{1} \bar{N} \times \bar{H}_{i n c}, 0,0$ for each surface observation point. The "Surface: Object" and "Object : Object" blocks are arranged so that $\hat{x}, \hat{y}$, and $\hat{z}$ components of (4) appear in succession for a given object observation point, while the unknown vector contains successive $J_{x}, J_{y}$, and $J_{z}$ values for a given object source point. These choices locate $1 / \alpha$ terms on the diagonal of the "Object: Object" matrix.
In many problems of interest, scattering from the rough interface can be much larger than that from the dielectric object (e.g., the case of a low contrast landmine buried in a lossy medium). In this case, a matrix equation in terms of object minus no-object difference fields can be formulated to clarify effects of the object. If the no-object problem is first solved through

$$
\text { [Surface : Surface }]\left[\bar{F}_{0}, \bar{G}_{0}\right]=\left[\bar{Q}_{i n c}\right]
$$

then the object minus no-object currents can be obtained from

$$
\begin{aligned}
& {\left[\begin{array}{c|c}
\text { Surface : Surface } & \text {-Object : Surface } \\
\text {-Surface : Object } & \text { Object : Object }
\end{array}\right]} \\
& \quad \cdot\left[\begin{array}{c}
\bar{F}-\bar{F}_{0}, \bar{G}-\bar{G}_{0} \\
\bar{J}
\end{array}\right] \\
& =\left[\begin{array}{c}
0 \\
\text { Surface : Object }\left\{\bar{F}_{0}, \bar{G}_{0}\right\}
\end{array}\right] .
\end{aligned}
$$

This differencing procedure is useful with an iterative solution of the matrix equation, since it insures that accuracy is retained in object scattering effects. The surface only solution is typically substantially less expensive than the combined surface/object problem (particularly with the canonical grid method described in Section IV) so that the additional computations are tolerable.

Consideration of object minus no object scattered fields also demonstrates an important issue in interpreting results for combined surface/object problems. Since object scattering is typically discussed in terms of radar cross sections while surface scattering is more appropriately described as a cross section per unit area, surface/object scattering problems include both standard and area extensive geometries making the definition of a radar cross section ambiguous. The results of this paper treat fields radiated in Region 1 by the difference currents $\Delta \bar{F}=$ $\bar{F}-\bar{F}_{0}, \Delta \bar{G}=\bar{G}-\bar{G}_{0}$ as equivalent to those radiated by an object in defining a radar cross section. However, since these difference currents contain object scattering but also interactions between the surface and object, a standard cross section is not obtained and results remain sensitive to the particular surface geometry and incident field considered.

\section{ITERATIVE METHODS}

Since the matrix equation (9) for 2-D surfaces and 3-D dielectric objects is likely to contain a large number of unknowns, direct inversion of (9) is computationally inefficient. Iterative solutions can be developed based on a multiple interaction procedure [6] or through an application of standard nonstationary iterative methods [15]. The former is accomplished for object minus no-object difference currents by iterating

$$
\begin{aligned}
& \text { [Object : Object] }\left[\bar{J}^{(n+1)}\right] \\
& \quad=\left[\text { Surface : Object }\left\{\bar{F}_{0}+\Delta \bar{F}^{(n)}, \bar{G}_{0}+\Delta \bar{G}^{(n)}\right\}\right] \\
& \text { [Surface : Surface] }\left[\Delta \bar{F}^{(n+1)}, \Delta \bar{G}^{(n+1)}\right] \\
& \quad=\left[\text { Object : Surface }\left\{\bar{J}^{(n+1)}\right\}\right]
\end{aligned}
$$


with the iteration initialized through $\Delta \bar{F}^{(0)}=0, \Delta \bar{G}^{(0)}=0$. The "Surface : Surface" and "Object: Object" matrix equations are solved with the nonstationary algorithm described below, with an initial guess for the $(n+1)$ th solution taken as the $n$th solution to improve convergence at each surface to object iteration. Since each iteration corresponds to an additional interaction between the surface and object, the method should be expected to converge rapidly for cases lacking strong surface/object interactions. Convergence can be monitored in terms of a "pseudo-residual" by examining the norms of $\Delta \bar{F}^{(n+1)}$ $\Delta \bar{F}^{(n)}$ and $\Delta \bar{G}^{(n+1)}-\Delta \bar{G}^{(n)}$.

Nonstationary methods applied to the combined object/surface matrix equation (11) can potentially provide improved convergence properties for cases with strong object/surface interactions and also converge in terms of the absolute residual of the matrix equation. The biconjugate gradient-stabilized algorithm (Bi-CGSTAB) was chosen for this paper, since it has been found effective in previous studies of rough surface scattering. Preconditioning can be beneficial with nonstationary methods if an approximation to the original matrix can be found which is easily inverted. This is accomplished for the matrix of (11) by neglecting surface/object coupling and employing an approximate solution of the "Surface: Surface" block based on a "quasi-physical optics" procedure as described in [21]. The "Object: Object" block of the preconditioner is solved through another Bi-CGSTAB routine set with only a moderate convergence accuracy. The effectiveness of this preconditioner is determined by the ratio of the number of surface and object unknowns; for cases with a much larger number of surface unknowns, the "Object:Object" solution is not expensive and the preconditioner is warranted. The matrix multiply acceleration methods described in the next Section further make the preconditioner effective. The results illustrated in Section V were computed using the combined object/surface matrix preconditioned $\mathrm{Bi}-\mathrm{CGSTAB}$ algorithm to insure that high accuracy was retained; limited tests with the surface/object iteration procedure of (12) and (13) showed good performance as well.

\section{CANONICAL GRID AND Discrete Dipole Algorithms}

To improve computational efficiency, the CAG [16]-[22] and DDA [24] methods are used to perform "Surface : Surface" and "Object:Object" block multiplies, respectively. Both algorithms are based on use of a uniform Cartesian grid for description of the surface profile (2-D grid) and dielectric object (three dimensional grid), and a Toeplitz representation for "Surface : Surface" and "Object : Object" matrices so that multiplies can be performed using the fast Fourier transform (FFT). Toeplitz matrices are obtained for the "Surface : Surface" block by expanding matrix elements in a series under the assumption of small height difference relative to the horizontal separation between points on the surface. The number of series terms retained is a parameter of the method, along with a "strong distance" between points inside of which the series expansion is not used. The method is most effective for small to moderate surface slopes and small to moderate surface heights in terms of $\lambda$, for which a small number of series terms and a small strong distance are needed. Other surface scattering acceleration techniques [14], [26] are likely to be more effective for large surface slopes and heights, but are unlikely to obtain the efficiency of the canonical grid method for small to moderate roughness surfaces. For the "Object: Object" block, the object is placed inside a uniform Cartesian grid and volumetric electric current sources set to zero in grid locations not occupied by the object. Three-dimensional FFT [27] computations involve the entire object grid, however, so the acceleration routine is most effective if the object occupies a significant fraction of the grid volume; elongated Cartesian grids can be used if necessary to insure that this occurs. Use of a uniform Cartesian grid can introduce "stair-stepping" errors in non-Cartesian boundaries, which are alleviated only through increased sampling rates. Reference [23] considers these errors for a spherically shaped object and shows that sampling rates from 10 to 20 points per wavelength in the object are acceptable. Both the canonical grid and discrete dipole methods provide $O\left(N_{s} \log N_{s}\right)$ and $O\left(N_{o} \log N_{o}\right)$ multiplies for "Surface: Surface" and "Object:Object" calculations, respectively, where $N_{s}$ is the number of surface unknowns and $N_{o}$ is the number of object unknowns.

Note that "Surface: Object" and "Object: Surface" coupling matrices are not accelerated in the current method, and therefore require $O\left(N_{s} N_{o}\right)$ computations. Development of FFT-based acceleration methods for these terms is complicated by the differing sampling requirements on $\bar{J}$ and $(\bar{F}, \bar{G})$ since differing dielectric media are involved. Use of FFT-based methods for sources and observation points on differing sampling rate grids would require careful consideration. Other matrix multiply acceleration methods can again avoid these problems. However, for problems in which $N_{s} \gg N_{o}$ acceleration of coupling matrix multiplies will provide only moderate gains in overall routine efficiency.

\section{SAMPLE APPLICATION: SCATTERING FROM A BURIED "LANDMINE"}

Fig. 2 illustrates a sample combined object/surface problem: a rectangular box dielectric object with dimensions $7.62 \mathrm{~cm} \times$ $7.62 \mathrm{~cm} \times 2.54 \mathrm{~cm}$ and relative permittivity $\epsilon_{3}=3+i 0.03$ is located $7.62 \mathrm{~cm}$ below an interface between free-space and a medium with relative permittivity $\epsilon_{2}=5+i 1.25$. Scattering from this object is to be determined for a field incident at $15^{\circ}$ from normal incidence at frequencies from 2 to $5.1 \mathrm{GHz}$. Results will be shown for both flat and rough interfaces between Regions 1 and 2. This geometry is intended to model ground penetrating radar observation of a low contrast (i.e., plastic) target in moderately attenuating soil; it is clear that object returns may be quite small when compared to potential interface scattering effects.

Fig. 2 also shows that a finite size interface (here, $1.28 \mathrm{~m} \times 1.28 \mathrm{~m}$ ) between Regions 1 and 2 is used in the model. To avoid artificial edge scattering effects due to this truncation, the problem is excited by the "tapered wave" incident field described in [17]. This field is designed to mimic an incident plane wave but provides attenuation of incident fields as surface edges are approached. For tapering parameter 


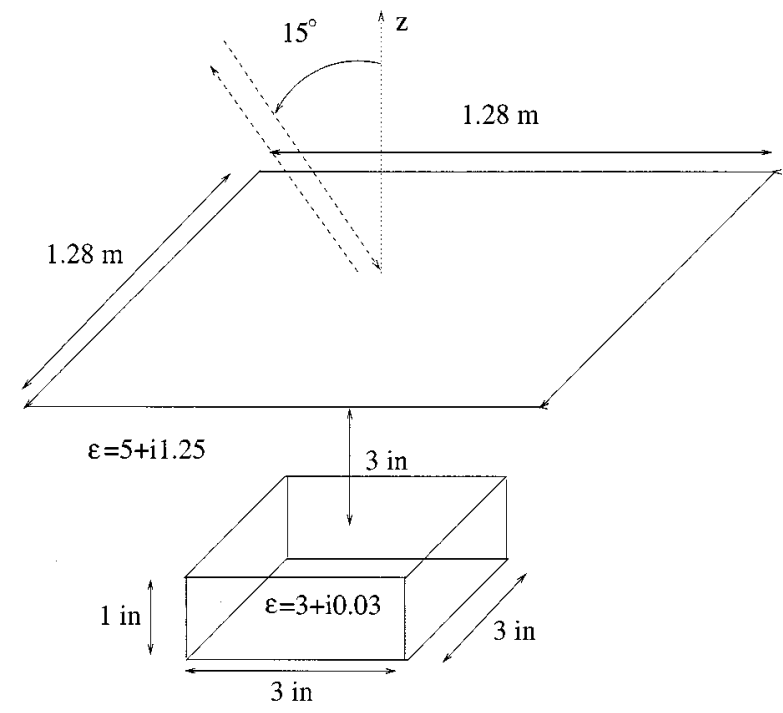

Fig. 2. "Landmine" detection geometry considered.

$g=5.333$, the field incident on surface edges is approximately $60 \mathrm{~dB}$ lower than that at the center, and the object horizontal cross section projected onto the flat interface is well within the 3-dB incident spot size. A test of tapered wave influence will be described below through comparison with a plane wave incidence Sommerfeld Green's function code in the flat surface limit.

\section{A. Computational Issues}

Since the $1.28 \mathrm{~m} \times 1.28 \mathrm{~m}$ interface ranges between 8.5 and 21.76 free-space wavelengths as the frequency is varied from 2 to $5.1 \mathrm{GHz}$, the interface is sampled into $128 \times 128$ points in the results shown. Although this is somewhat small for the higher frequencies given that the lower medium wavelength is approximately 2.25 times shorter than that in free-space, a set of comparisons using $256 \times 256$ points showed only slight changes in scattered cross sections. While a smaller number of points could be used for the lower frequencies, a constant number of points sampling the interface as frequency is varied was chosen for convenience in describing the rough surface profile. The resulting number of field unknowns on the interface is 65536 . The object is sampled on a $32 \times 32 \times 32$ point grid with step size $3.175 \mathrm{~mm}$ (ranging from approximately $1 / 27$ to $1 / 11$ of the wavelength in the object as frequency varies), so that the object occupies $14 \%$ of the grid volume; again this choice was made for simplicity in initial tests but more effective grids (e.g., a $32 \times 32 \times 8$ point grid) could be used. The total number of unknowns for the object is then 13824 , and the combined problem contains 79360 unknowns.

Although the problem considered can be solved on a PC level platform, total computing times for the multiple cases considered in this paper were further reduced through use of IBM SP parallel computing resources at the Maui High Performance Computing Center [28]. Since results as a function of frequency were of interest, single frequency calculations were performed on individual nodes of the parallel computer (comparable to $\mathrm{PC}$ platforms) to obtain 32 frequencies between 2 and $5.1 \mathrm{GHz}$. Single frequency computing times on a single

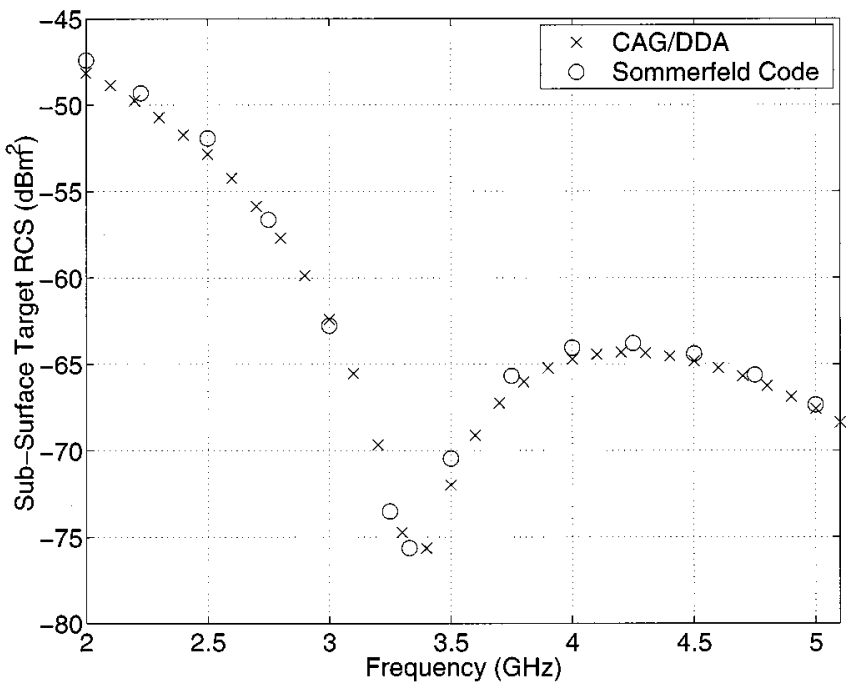

Fig. 3. Object minus no object radar cross sections for geometry of Fig. 2 versus frequency, $H H$ polarization. Comparison of CAG/DDA method with a Sommerfeld Green's function-based solution.

node ranged from approximately one to eight hours depending on convergence parameters and the iterative method used; attempts to optimize computing times have at present not been performed extensively.

\section{B. Validation}

To validate the CAG/DDA model, backscattered radar cross sections from the subsurface object were compared with those obtained by a Sommerfeld Green's function-based code [29] in the flat surface limit. Fig. 3 illustrates the comparison versus frequency for $H H$ polarization, and shows good agreement between the CAG/DDA and Sommerfeld codes even through a relatively large variation in cross sections. Small cross section values observed between 3 and $3.5 \mathrm{GHz}$ can be explained due to interference between reflections from the top and bottom interfaces of the target, as predicted through a simple multilayer reflection coefficient model. Some discrepancies within $1 \mathrm{~dB}$ are observed, but are difficult to resolve due to computational limitations of the Sommerfeld code which is based on direct matrix equation solution. The good agreement also confirms that the tapered wave incident field used in the CAG/DDA model is effectively modeling a plane wave incident field while reducing surface edge scattering effects.

\section{Effect of a Slight Surface Roughness}

Fig. 4 repeats the results of Fig. 3 but includes a slight surface roughness on the interface between Regions 1 and 2 . A Gaussian random process description of $f(x, y)$ was used with an isotropic Gaussian correlation function. A surface height standard deviation of $3.58 \mathrm{~mm}$ and correlation length of $3.58 \mathrm{~cm}$ were chosen; this height standard deviation ranges from approximately $1 / 42$ to $1 / 17$ of the free-space wavelength so that the roughness exceeds limitations of the analytical small perturbation method (SPM) [30] for rough surface scattering only at the highest frequencies. Two terms in the canonical grid series and a "strong distance" of 15 points were used to insure accurate inclusion of roughness effects in "Surface: Surface" 


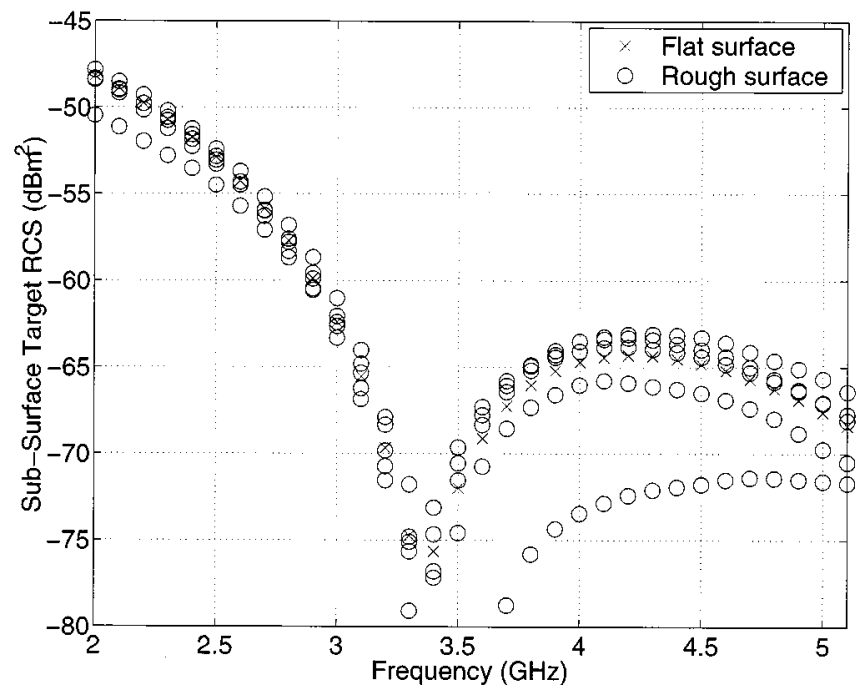

Fig. 4. Effect of surface roughness on object minus no object radar cross sections versus frequency, $H H$ polarization. Results are illustrated for five surface realizations.

coupling. Results were calculated for five distinct realizations of the surface random process and object minus no object "radar cross sections" are compared with the flat surface case in Fig. 4. The presence of surface roughness is observed to have only a moderate influence on object backscattering versus frequency, with larger effects obtained at the higher frequencies where roughness is larger in terms of the wavelength. Although further calculations could be performed to obtain a Monte Carlo averaged object RCS in the presence of the specified random process, the single realization results illustrated are also of interest since they also give some idea as to the level of variations that may be observed for a given surface profile.

An additional validation of surface scattering contributions can be performed for the problem considered through comparison of surface only scattering with the SPM. Fig. 5 shows the comparison of in-plane $H H$ and $V V$ incoherent bistatic radar cross sections (scattering angle $-15^{\circ}$ indicates backscattering) at $3 \mathrm{GHz}$ averaged over 32 surface realizations. Results are plotted in terms of absolute cross sections rather than cross sections per unit area: SPM predictions are simply scaled by the area illuminated by the tapered incident field to obtain the curves shown. Note the much larger surface scattering cross sections compared to those of the object in Fig. 4; the low contrast target and moderate attenuation in Region 2 produce this effect, even with the slight surface roughness considered. Some discrepancies between CAG/DDA and SPM predictions near the forward scattering region are observed due to inaccuracies in subtraction of the coherent forward scattered tapered wave that exists for this slight surface roughness. Overall, however, the good agreement obtained validates the CAG/DDA method developed for calculation of both object and surface scattering.

A final illustration of combined object/surface scattering effects is provided in Fig. 6, which plots the envelope of time domain backscattered fields obtained from 2 to $5.1 \mathrm{GHz}$ data for the flat surface case and one of the rough surfaces. Object scattering cross sections are observed to return later in time, as expected, and again to have significantly lower

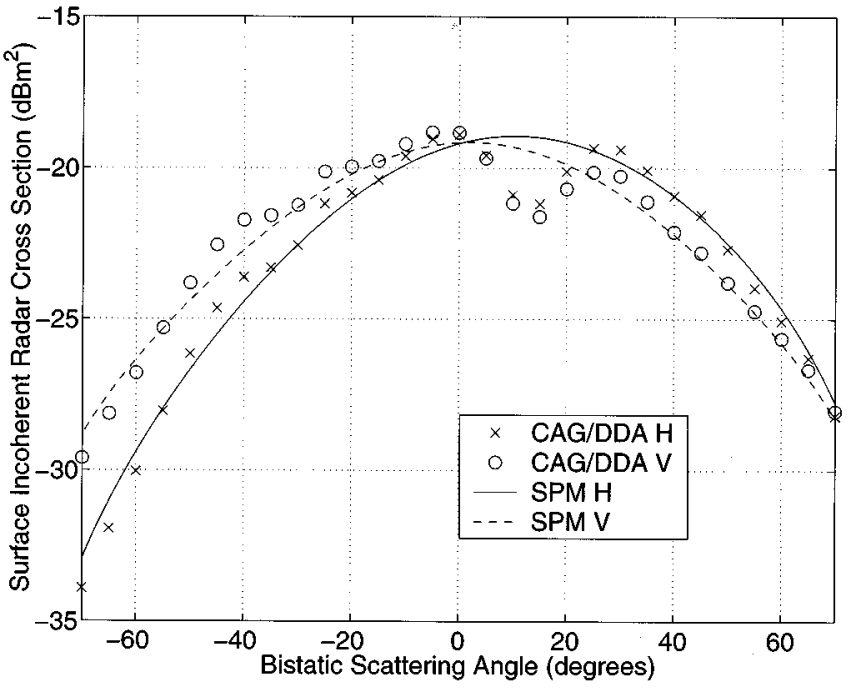

Fig. 5. Average in-plane incoherent surface bistatic scattering cross sections. SPM results are included for comparison.

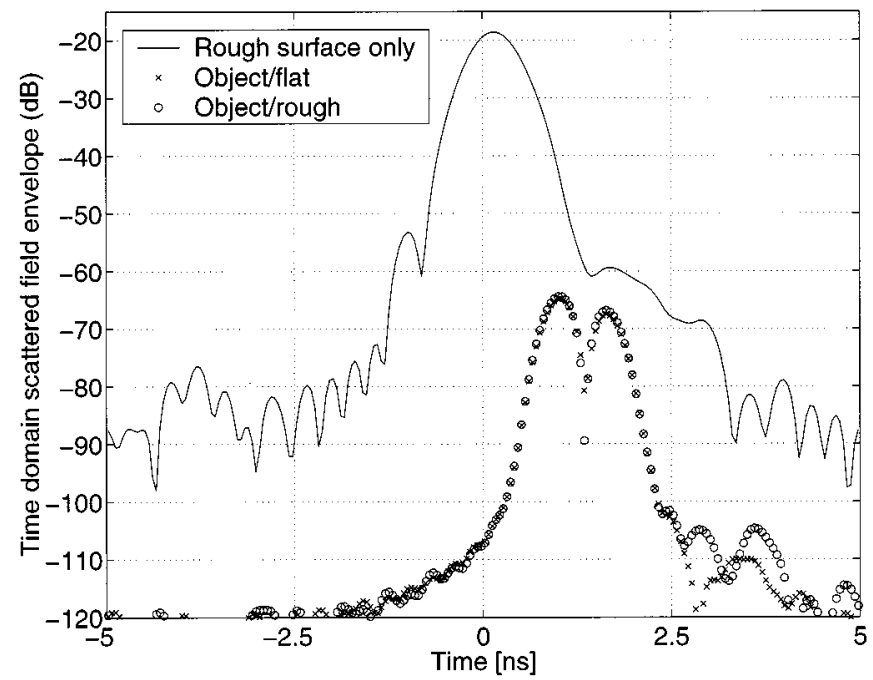

Fig. 6. Envelope of time domain $H H$ backscattered fields for geometry of Fig. 2.

amplitudes than rough surface scattering contributions. Surface roughness effects are observed to have only a moderate effect on time domain object cross sections; results with other surface realizations show similar levels of differences. The comparison of Fig. 6 demonstrates the typical problem of small object scattering in the presence of much larger surface clutter for ground penetrating radar systems. Effective signal processing algorithms are thus required to detect these objects.

\section{CONCLUSIONS}

The CAG/DDA model presented makes numerical calculation of combined surface/dielectric object geometries relatively efficient, and can be applied to many areas of current interest. Further improvements of the method can be obtained through acceleration of "Surface : Object" and "Object: Surface" multiplies with FFT-based methods or other techniques, and also through further tests to optimize parameter choices for the algorithm. The results shown illustrate the importance of including 
both object and surface scattering contributions. Evaluations of analytical models for surface/object interactions [1]-[4] are also possible with the method, and insights obtained from further studies should enable improved analytical models to be created.

\section{REFERENCES}

[1] Y. Zhang, Y. E. Yang, H. Braunisch, and J. A. Kong, "Electromagnetic wave interaction of conducting object with rough surface by hybrid SPM/MOM technique," PIER 22: Progr. Electromagn. Res., vol. 22, pp. 315-335, 1999.

[2] T. Chiu and K. Sarabandi, "Electromagnetic scattering interaction between a dielectric cylinder and a slightly rough surface," IEEE Trans. Antennas. Propagat., vol. 47, pp. 902-913, May 1999.

[3] J. T. Johnson, "Thermal emission from a layered medium bounded by a slightly rough interface," IEEE Trans. Geosci. Remote Sensing, vol. 39, pp. 368-378, Feb. 2001.

[4] I. M. Fuks and A. G. Voronovich, "Wave diffraction by rough interfaces in an arbitrary plane-layered medium," Waves Random Media, vol. 10, pp. 253-272, 2000.

[5] Proc. SPIE 4098: Detection and Remediation Technologies for Mines and Minelike Targets V, A. C. Dubey et al., Eds., 2000.

[6] G. F. Zhang, L. Tsang, and K. Pak, "Angular correlation function and scattering coefficient of electromagnetic waves scattered by a buried object under a two-dimensional rough surface," J. Opt. Soc. Amer. A, vol. 15, pp. 2995-3002, 1998.

[7] J. Q. He, T. J. Yu, N. Geng, and L. Carin, "Method of moments analysis of electromagnetic scattering from a general three-dimensional dielectric target embedded in a multilayered medium," Radio Sci., vol. 32, pp. 305-313, 2000

[8] T. J. Cui and W. C. Chew, "Fast algorithm for electromagnetic scattering by buried 3-D dielectric objects of large size," IEEE Trans. Geosci. Remote Sensing, vol. 37, pp. 2597-2608, Sept. 1999.

[9] S. Tjuatja, A. K. Fung, and J. Bredow, "Radar imaging of buried objects," in Proc. Int. Geoscience and Remote Sensing Soc., 1998, pp. 524-526.

[10] K. O’Neill, R. F. Lussky, and K. D. Paulsen, "Scattering from a metallic object embedded near the randomly rough surface of a lossy dielectric," IEEE Trans. Geosci. Remote Sensing, vol. 34, pp. 367-376, 1996.

[11] T. Dogaru and L. Carin, "Time-domain sensing of targets buried under a rough air-ground interface," IEEE Trans. Antennas Propagat., vol. 46, pp. 360-372, Mar. 1998.

[12] A. V. der Merwe and I. J. Gupta, "A novel signal processing technique for clutter reduction in GPR measurements of small, shallow land mines," IEEE Trans. Geosci. Remote Sensing, vol. 38, pp. 2627-2637, Nov. 2000.

[13] M. R. Pino, L. Landesa, J. L. Rodriguez, F. Obelleiro, and R. J. Burkholder, "The generalized forward-backward method for analyzing the scattering from targets on ocean-like rough surfaces," IEEE Trans. Antennas Propagat., vol. 47, pp. 961-969, 1999.

[14] M. El-Shenawee and C. Rappaport, "Modeling clutter from random rough ground for GPR subsurface sensing applications," in Proc. PIERS, Cambridge, MA, 2000, p. 39.

[15] R. Barrett, M. Berry, T. Chan, J. Demmel, J. Donato, J. Dongarra, V. Eijkhout, R. Pozo, C. Romine, and H. van der Vorst, Templates for the Solution of Linear Systems: Building Blocks for Iterative Methods. Philadelphia, PA: SIAM, 1993.

[16] L. Tsang, C. H. Chan, K. Pak, and H. Sangani, "Monte Carlo simulations of large scale problems of random rough surface scattering and applications to grazing incidence with the BMIA/canonical grid method," IEEE Trans. Antennas Propagat., vol. 43, pp. 851-859, Aug. 1995.

[17] K. Pak, L. Tsang, C. H. Chan, and J. T. Johnson, "Backscattering enhancement of electromagnetic waves from two dimensional perfectly conducting random rough surfaces based on Monte Carlo simulations," J. Opt. Soc. Amer. A, vol. 12, pp. 2491-2499, 1995.

[18] K. Pak, L. Tsang, and J. T. Johnson, "Numerical simulations and backscattering enhancement of electromagnetic waves from two dimensional dielectric random rough surfaces with sparse matrix canonical grid method," J. Opt. Soc. Amer. A, vol. 14, pp. 1515-1529, 1997.
[19] J. T. Johnson, R. T. Shin, J. A. Kong, L. Tsang, and K. Pak, "A numerical study of the composite surface model for ocean scattering," IEEE Trans. Geosci. Remote Sensing, vol. 36, pp. 72-83, Jan. 1998.

[20] J. T. Johnson and H. T. Chou, "Numerical studies of low grazing angle backscatter from 1-D and 2-D impedance surfaces," in Proc. Int. Geoscience and Remote Sensing Soc., vol. 4, 1998, pp. 2295-2297.

[21] J. T. Johnson, "A numerical study of low grazing angle backscatter from ocean-like impedance surfaces with the canonical grid method," IEEE Trans. Antennas Propagat., vol. 46, pp. 114-120, 1998.

[22] — "On the canonical grid method for two dimensional scattering problems," IEEE Trans. Antennas Propagat., vol. 46, pp. 297-302, 1998.

[23] B. T. Draine and P. J. Flatau, "Discrete-dipole approximation for scattering calculations," J. Opt. Soc. Amer. A, vol. 11, pp. 1491-1499, 1994.

[24] P. J. Flatau, "Improvements in the discrete-dipole approximation method of computing scattering and absorption," Opt. Lett., vol. 22, pp. 1205-1207, 1997.

[25] J. T. Johnson, R. T. Shin, J. A. Kong, L. Tsang, and K. Pak, "A numerical study of ocean polarimetric thermal emission," IEEE Trans. Geosci. Remote Sensing, vol. 37, pp. 8-20, Jan. 1999.

[26] H. T. Chou and J. T. Johnson, "A novel acceleration algorithm for the computation of scattering from rough surfaces with the forward-backward method," Radio Sci., vol. 33, no. 5, pp. 1277-1287, 1998.

[27] M. Frigo and S. G. Johnson. FFTW 2.1.3 Manual. [Online]. Available: www.fftw.org.

[28] Maui high performance computing center world wide web site. [Online]. Available: www.mhpcc.edu

[29] E. Newman, "A user's manual for the electromagnetic surface patch code: Preliminary version ESP5.0," ElectroScience Lab., The Ohio State Univ., Columbus, 1997.

[30] S. O. Rice, "Reflection of electromagnetic waves from slightly rough surfaces," Commun. Pure Appl. Math., vol. 4, pp. 361-378, 1951.

Joel T. Johnson (M'96) received the B.S. degree in electrical engineering from the Georgia Institute of Technology, Atlanta, in 1991 and the S.M. and Ph.D. degrees from the Massachusetts Institute of Technology (MIT), Cambridge, in 1993 and 1996, respectively.

He is currently an Associate Professor in the Department of Electrical Engineering and ElectroScience Laboratory, The Ohio State University (OSU), Columbus. His research interests are in the areas of microwave remote sensing, propagation, and electromagnetic wave theory.

Dr. Johnson is an Associate Member of Commissions B and F of the International Union of Radio Science (URSI), and a Member of Tau Beta Pi, Eta Kappa $\mathrm{Nu}$, and Phi Kappa Phi. He received the 1993 Best Paper Award from the IEEE Geoscience and Remote Sensing Society, and was named an Office of Naval Research Young Investigator, National Science Foundation Career Awardee, and PECASE Award Recipient in 1997.

Robert J. Burkholder (S'85-M'89-SM'97) received the B.S., M.S., and $\mathrm{Ph} . \mathrm{D}$. degrees in electrical engineering from The Ohio State University (OSU), Columbus, in 1984, 1985, and 1989, respectively.

Since 1989, he has been with the OSU ElectroScience Laboratory, Department of Electrical Engineering, where he is currently Research Scientist and Adjunct Associate Professor. His research specialties are high-frequency asymptotic techniques and their hybrid combination with numerical techniques for solving large-scale electromagnetic radiation and scattering problems. He has contributed extensively to the EM analysis of large cavities, such as jet inlets/exhausts, and is currently working on the more general problem of EM radiation, propagation, and scattering in realistically complex environments.

Dr. Burkholder is a Full Member of URSI Commission B, and a Member of the Applied Computational Electromagnetics Society (ACES). 